\title{
Towards a Reformulated Theory Underlying Schema Therapy: Position Paper of an International Workgroup
}

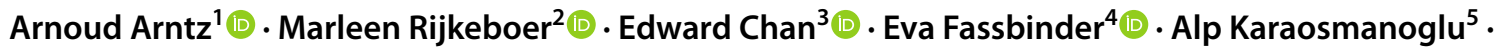 \\ Christopher William Lee ${ }^{6,7} \cdot$ Marta Panzeri $^{8}(\mathbb{D}$
}

Accepted: 16 January 2021 / Published online: 9 February 2021

(c) The Author(s) 2021

\begin{abstract}
Background A central construct in Schema Therapy (ST) is that of a schema mode, describing the current emotional-cognitive-behavioral state. Initially, 10 modes were described. Over time, with the world-wide increasing and broader application of ST to various disorders, additional schema modes were identified, mainly based on clinical impressions. Thus, the need for a new, theoretically based, cross-cultural taxonomy of modes emerged.

Methods An international workgroup started from scratch to identify an extensive taxonomy of modes, based on (a) extending the theory underlying ST with new insights on needs, and (b) recent research on ST theory supporting that modes represent combinations of activated schemas and coping.

Results We propose to add two emotional needs to the original five core needs that theoretically underpin the development of early maladaptive schemas (EMSs), i.e., the need for Self-Coherence, and the need for Fairness, leading to three new EMSs, i.e. Lack of a Coherent Identity, Lack of a Meaningful World, and Unfairness. When rethinking the purpose behind the different ways of coping with EMS-activation, we came up with new labels for two of those: Resignation instead of Surrender, and Inversion instead of Overcompensation. By systematically combining EMSs and ways of coping we derived a set of schema modes that can be empirically tested.
\end{abstract}

Conclusions With this project, we hope to contribute to the further development of ST and its application across the world.

Keywords Schema therapy $\cdot$ Early maladaptive schemas $\cdot$ Schema modes $\cdot$ Needs $\cdot$ Personality disorders

\section{Introduction}

In the last two decades Schema Therapy (ST) was found highly effective and became increasingly popular as a treatment of chronic psychopathology, including personality disorders, chronic depression, and severe eating disorders,

Arnoud Arntz and Marleen Rijkeboer shared first authorship.

Marleen Rijkeboer

marleen.rijkeboer@maastrichtuniversity.nl

1 Department of Clinical Psychology, University of Amsterdam, Amsterdam, the Netherlands

2 Department of Clinical Psychological Science, Maastricht University, P.O. Box 616, 6200 MD Maastricht, the Netherlands

3 International Psychology Centre, International Psychology \& Complementary Medicine University, Kuala Lumpur, Malaysia

4 Department of Psychiatry and Psychotherapy, University of Lübeck, Lübeck, Germany
5 Psikonet, Psychotherapy and Training Center, Istanbul, Turkey

6 Discipline of Psychology, College of Science, Health, Engineering and Education, Murdoch University, Perth, WA, Australia

7 Faculty of Health and Medical Sciences, The University of Western Australia, Crawley, WA, Australia

8 Department of Developmental Psychology and Socialisation, University of Padua, Padua, Italy 
and it was disseminated rapidly in many cultures all over the world (Arntz and Jacob 2012; Arntz and van Genderen 2009; Chan and Tan 2020; Jacob and Arntz 2013; Rafaeli et al. 2010; Renner et al. 2013; Sempérteguia et al. 2013; Simpson et al. 2010; Young et al. 2003). ST was formulated in the late former century by Jeffrey Young as an extension of cognitive-behavioral therapy (CBT), integrating elements from different schools (e.g., experiential and psychodynamic therapies), the attachment literature, and other developmental theories into a coherent theory (Young 1994; Young et al. 2003). Since then ST has evolved considerably, including e.g., the advancement of the mode construct, and the development of specific modes for borderline personality disorder (Lobbestael et al. 2007, 2008, 2010; Young et al. 2007), cluster C personality disorders (Bamelis et al. 2011), and forensic psychopathology (Keulen de Vos et al. 2016,2017). However, a clear theoretical framework that steers the addition of these concepts is lacking. Moreover, although ST is used worldwide, it remains unclear whether these concepts are equally valid across cultures (cf., Hofmann 2006). For example, ST was developed in the Western culture where people tend to have more independent self-construals, whereas non-Western people have interdependent self-construals (Ayyash-Abdo et al. 2016). Hence, an international workgroup, consisting of the authors of this paper who are from various cultural backgrounds, reviewed the fundaments of the theory underlying ST, leading to a reformulation of this theory. The workgroup's endeavors are laid down in the present position paper.

\section{Theory Underlying ST as Originally Formulated by Young and Colleagues}

\section{Origin of Early Maladaptive Schemas}

At first, the focus of ST was mostly on early maladaptive schemas (EMSs), i.e., dysfunctional mental representations that are hypothesized to be formed during early development as the result of an interaction between temperamental factors in the child and adverse environmental factors, such as abuse, neglect, or dysfunctional parenting. In short, in ST, chronic (or characterological) psychopathology is thought to result when universal emotional needs are not adequately fulfilled during childhood. Young et al. (2003) hypothesized five emotional needs to be essential for healthy development, i.e., (1) safety \& nurturance (including safe attachment); (2) autonomy, competence, \& sense of identity; (3) freedom to express needs, opinions, \& emotions; (4) spontaneity \& play, and (5) realistic limits \& self-control. When these needs are not met, there is an increased risk that the child forms maladaptive schemas about the self, others, and the world, that interfere with healthy adaptation later in life. Eighteen schemas have been formulated and grouped into five domains, which correspond to the aforementioned hypothesized needs (see Table 1).

The theory further states that specific stimuli can activate EMSs, and when fully activated these will dominate the feeling and information processing of the person. Behavioral responses are not hypothesized to be part of the EMSs, but rather follow EMS activation.

\section{Coping with EMS Activation}

People are thought to develop ways to deal with actual or impending/potential EMS activation. Young described three ways of coping with EMS activation: (1) surrender (giving in to the EMS activation, accepting the EMS as if it is true); (2) avoidance (avoiding or escaping EMS activation by mental or behavioral responses); (3) overcompensation (the person fights the EMS by believing the opposite of the EMS is true, and feels and behaves accordingly). Note, that "coping" here denotes how the person deals with an internal (mental) experience, the (threat of) activation of an EMS, and is not about how the person deals with external circumstances. Moreover, according

Table 1 Overview of needs (original and additional) and the respective Early Maladaptive Schemas (EMSs)

\begin{tabular}{ll}
\hline Needs & Respective EMSs \\
\hline Original & Original \\
Safety \& nurturance & Emotional deprivation \\
& Mistrust/abuse \\
& Abandonment \\
& Social isolation \\
& Defectiveness/shame \\
Autonomy, competence, \& identity & Dependence/incompetence \\
& Failure \\
& Vulnerability to harm \& illness \\
& Enmeshment \\
Freedom to express needs, opinions, & Subjugation \\
\& emotions & Self-sacrifice \\
& Approval seeking \\
Spontaneity \& play & Negativity/pessimism \\
Fairness & Emotional inhibition \\
Realistic limits \& self-control & Unrelenting standards \\
Additional & Punitiveness \\
& Entitlement/grandiosity \\
& Insufficient self-control/self- \\
& discipline \\
& Additional \\
& Lack of a coherent identity \\
& Lack of a meaningful world \\
& Unfairness \\
&
\end{tabular}


to the original theory coping responses are generally automatic and people are not necessarily making a conscious decision for them.

\section{Schema Modes}

Coping responses to EMSs result in so-called schema modes, which describe the momentary emotional-cognitive-behavioral state of the person, whereas EMSs are more trait like. Furthermore, in contrast to EMSs, schema modes include behavior and are more directly connected to the problems (symptoms) of the patient.

Young et al. (2003) proposed three types of dysfunctional modes. Firstly, they identified child modes, which are strongly related to EMSs, and in which the person feels, thinks, and behaves as a child. Secondly, internalized parent modes were distinguished, describing states in which the person experiences excessive self-punishment or extreme demands for achievement or high standards. These are hypothesized to result from the internalization of dysfunctional moral standards and related behavior of caregivers. Thirdly, there are coping modes, which denote the state of being in which attempts to escape or compensate the EMS dominate. Examples are the Detached Protector (feeling nothing as the result of detaching from emotions and needs) and the Self-Aggrandizer (aggrandizing one's importance to compensate for an EMS with opposite implications). Note, that all dysfunctional modes are a combination of an activated EMS and a way of coping; in the child modes the individual "acquiesces" to the EMS activation, hence intense feelings that are tied to a specific EMS dominate, whereas in the "coping" modes the individual tries to avoid or "invert" its contents, and thus behaviors and thoughts that aim to undo the pain caused by a specific EMS dominate. Lastly, Young also identified two functional modes, the Happy Child and the Healthy Adult Mode.

Note, that the same EMS can underlie different types of problems. Which of these problems is expressed depends on the type of schema mode(s) that are typically activated in the person. For instance, the same EMS from the disconnection domain (e.g., Abandonment) can underlie both internalizing (e.g., abandonment depression) and externalizing forms of psychopathology (e.g., aggressively threatening the other who is abandoning the person), depending on the way of coping (see Wijk-Herbrink et al. 2018a, b, for an empirical test). Likewise, the same problem or symptom can result from different EMSs, i.e., a symptom might be determined by "surrender" to one schema, or by "overcompensation" for another schema. For example, perfectionism as a symptom can be the result of surrendering to a schema of Unrelenting Standards and/or overcompensation for a Failure schema.

\section{Developments in Schema Therapy}

Modern approaches to ST are based on the schema mode model. Schema modes are central to the application of ST especially with more severe patients, as they help clarify the state the person is in, as well as the (often sudden) switches patients make between such states. Moreover, current ST protocols describe specific techniques to be applied for specific modes. Research has corroborated that both patients and therapists acknowledge the usefulness of the schema mode model in the treatment process, helping to form a (meta-cognitive) model of the patient's problems and steering the treatment process (de Klerk et al. 2017; Tan et al. 2018).

Given the usefulness of the schema mode model, there is a growing need amongst clinicians and researchers worldwide for a cross-cultural, comprehensive taxonomy of modes. To enable a formulation of such a taxonomy, the theoretical foundations of schema therapy were critically evaluated by the workgroup, and described in this position paper. We start with a discussion of the core emotional needs in early life, that underlie the development of EMSs. There is an extant literature on fundamental human needs, synthesized in several reviews (e.g., Baumeister and Leary 1995; Deci and Ryan 2000; Pittman and Zeigler 2007). Dweck (2017) recently elaborated on existing research, and developed a modern comprehensive theory of emotional needs, integrating motivation, personality, and (social-cultural) psychological development, hence unifying aspects of earlier frameworks of needs. Dweck's theory aligns with Young's ideas of personality development, but it is far more elaborated and scientifically grounded. Therefore, we chose to start with Dweck's framework as a reference point for evaluating the theoretical fundaments of schema therapy. Next, we discuss a fundamental need that might have been overlooked both in ST theory and in Dweck's framework. Then we discuss the nature of coping in Young's model and the confusion surrounding these concepts, followed by the theoretical guidelines for constructing a comprehensive set of modes. Lastly, we propose a set of schema modes based on this renewed formulation of ST theory, and discuss how the new formulation can be empirically tested.

\section{Evaluation and Reformulation of Young's Theory of Needs}

\section{Young's and Dweck's Needs Theories Compared}

As said, Young described five core emotional needs, while acknowledging that these were not based on a comprehensive theory and that the set of needs he proposed might be incomplete (Young et al. 2003). Similar to Young et al. 
(2003), Dweck's theory (2017) described how core emotional needs are important in the development of personality, using similar constructs as schemas (i.e., mental representations, called BEATs, which stands for beliefs, emotions, and action tendencies) and schema modes (called online acts and experiences). Dweck postulated seven evidence-based emotional needs (Fig. 1), and discussed empirical evidence for each of them. In short, she argued that three emotional needs are most basic in development: acceptance, predictability, and competence. The need for acceptance represents the need for positive social engagement, and overlaps with similar constructs proposed in the literature, such as connectedness, attachment, affiliation, belonging, or love (Dweck 2017; see p. 691). This need is proposed to be present very early in development. The second primary need, i.e., the need for (optimal) predictability, stands for "the desire to know the relationships among events and among things in the world: what follows what, what belongs with what, or what causes what" (Dweck 2017, p. 692). The third primary need according to Dweck is the need for competence, which is the need to build "skills for acting in or on the world" (Dweck 2017, p. 692). Resting on empirical foundations, Dweck argued that the aforementioned needs are the earliest developmentally and therefore are the most basic, i.e., they appear starting in infancy, guide both goal-directed behavior and information processing even at that early stage, and when thwarted lead to serious risk and a failure to thrive.

She goes on to argue that based on these three most basic needs four 'compound needs' develop: the needs for trust, control, self-esteem/status, and self-coherence. Each of the first three evolve from the combination of a pair of basic needs. In particular, the need for trust starts to evolve from 7 to 9 months out of the combined needs for acceptance and predictability. According to Dweck, these two basic needs are distinct until that age, and do not join into what we would call trust. Similarly, the need for control evolves from the combined needs for competence and predictability, and corresponds to what others called the need for agency, autonomy, and self-control. It represents the need to manipulate the world, for which predictability of the world and competence in the necessary skills are a prerequisite. Finally, the need for self-esteem/status gradually unfolds in the second year of development out of a conjunction of the needs for acceptance and competence. According to Dweck (2017, p. 294), "the outcomes of both acceptance-related goals and competence-related goals provide information about one's merits and standing". The reason why these compound needs evolve later in time than the three basic needs is related to the necessary cognitive and behavioral capacities that have to develop first.

The fourth and final compound need identified by Dweck, namely the need for self-coherence, stands for the desire to feel psychologically intact and rooted. It is about the need for experiencing the self as integrated and the world as meaningful, in relation to the person. These two sub-aspects are called identity and meaning. This need is fulfilled by the successful integration of the areas of the six other needs.

We tried to locate the five emotional needs described by Young et al. (2003) within Dweck's taxonomy, and constructed a map showing the relationship between the two (see Fig. 1). Below we present how each of the needs identified by Young maps onto Dweck's system.

1. Safety \& nurturance (including safe attachment). This need covers the innate need of the child for support and acceptance by caregivers (e.g., to be calmed when in
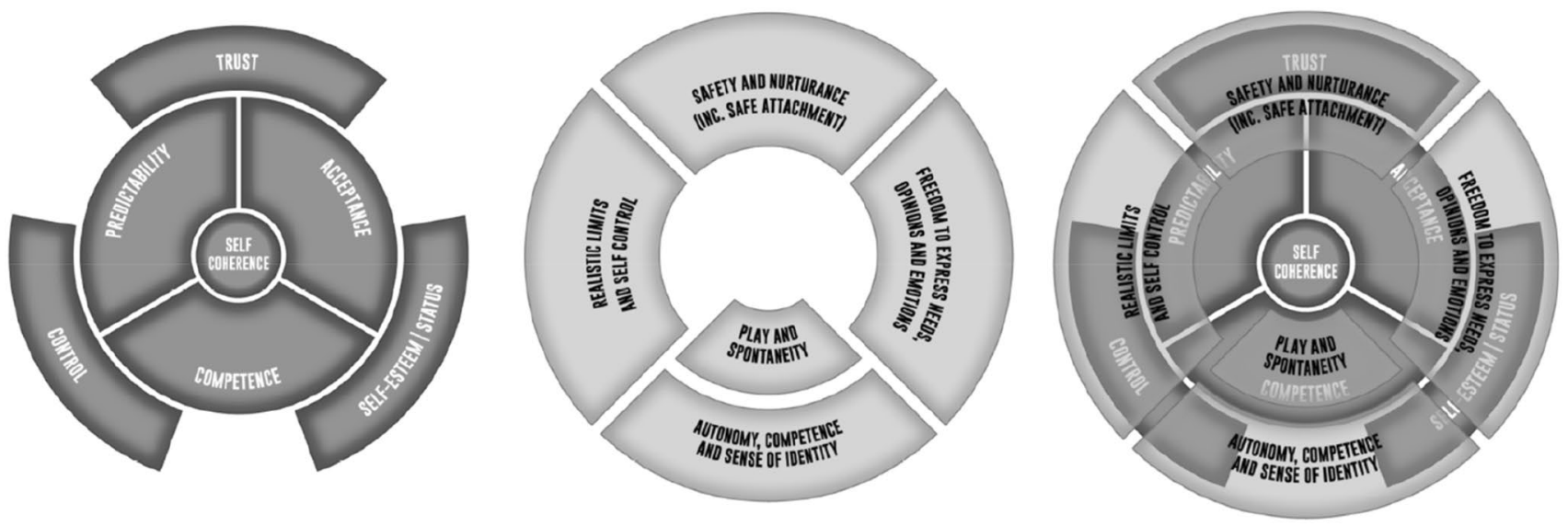

Fig. 1 Young's five emotional needs mapped onto Dweck's seven needs. Left: Dweck's seven needs: The three basic needs of acceptance, predictability, and competence; the compound or emergent needs of trust, control, and self-esteem/status; and a seventh emergent need of self-coherence at the intersection of all the other needs (freely adapted from Dweck (2017)). Middle: Young's five core emotional needs. Right: Young's needs mapped onto Dweck's needs. Note that Dweck's superordinate need for self-coherence is not covered by Young's needs 
stress) and a predictable environment, in which trust for caregivers and the environment develops. When support and acceptance are not met, the child may develop the EMSs "emotional deprivation", "social isolation", and/or "defectiveness/shame"; when no predictable environment is given, the EMSs "abandonment" and/ or "mistrust/abuse" might develop. Therefore, this need overlaps with the domain of Dweck's needs of acceptance, predictability, and trust.

2. Autonomy, competence \& sense of identity. This need is about developing a sense of being able to make independent decisions, discovering the world, being able to overcome problems, and forming a sense of identity as an independent person with specific competencies. This overlaps with the area in Dweck's system where competence, control, and self-esteem/status lie.

3. Freedom to express needs, opinions \& emotions. In order to be allowed to express needs, opinions, and emotions, the child has to expect that this will be accepted. Also, the child has to have a sense of competence that it is able to verbalize these. Lastly, the child has to experience a certain status and self-esteem to feel socially allowed to express needs, opinions, and emotions-and conversely, expressing these will build up self-esteem and status. Hence, this need overlaps with the domain in Dweck's taxonomy defined by acceptance, competence, and selfesteem/status.

4. Spontaneity \& play. Both in humans and animals, playing is a way to develop competence. The need for spontaneity and play can therefore be placed in the area of Dweck's need for competence.

5. Realistic limits \& self-control. Without realistic limits, the environment of the child becomes unpredictable and uncontrollable. Moreover, realistic limits offer learning experiences in competence and control. This need can therefore be placed in the area of predictability, competence, and control in Dweck's taxonomy.

As can be seen, Young's list overlaps nicely with Dweck's taxonomy. However, Young's overview of needs lacks the need for self-coherence, which incorporates the two distinguishable aspects identity ("Who am I?") and meaning ("How does/should the world work in ways that matter to me?"; Dweck 2017, p. 695). One could think that Young's need for autonomy, competence, and sense of identity includes the need for self-coherence. However, the identity aspect of the former need relates more to experiencing identity through achievement and competence ("I know a lot about dinosaurs, I love climbing trees, I am good at soccer, etc.”) than experiencing self-coherence. Apart from the need for self-coherence, Young's list of emotional needs thus appears to cover the emotional needs of humans as defined by Dweck well. With the exception of self-coherence, we can therefore assume that the Early Maladaptive Schemas (EMSs) that are derived from these needs represent the fundamental maladaptive representations in humans well, and therefore a systematic derivation of schema modes from the combinations of EMSs and coping will lead to good coverage of the universe of modes.

\section{The Need for Self-Coherence and Related EMSs}

Because the need for self-coherence is missing in Young's taxonomy of needs, we propose that this need (with its two aspects) and the related EMSs of lack of a coherent identity, and lack of a meaningful world should be added.

Lack of a coherent identity refers to the representation of the self as non-coherent and diffuse, as consisting out of non-integrated parts, and in severe cases as consisting out of completely dissociated parts. Lack of a meaningful world refers to the representation of the world as meaningless, with the self being disconnected from the processes taking place in the world. Activation of these schemas will lead to feelings of confusion, estrangement, existential anxiety, the self and/or the world falling apart, being lost, et cetera. As experiences cannot be integrated into a meaningful whole, symptoms of dissociation and psychosis might result. Thus, we expect high levels of these EMSs in severe psychopathologies, for example in severe personality disorders (Borderline (identity diffusion), Schizoid, Schizotypal), Dissociative Identity Disorder, and severe (chronic) psychosis. For example, in Schizoid Personality Disorder, although one can see one's own life as meaningful and the self as integrated, there is an estrangement from the world around, as is presumably reflected by a high level of the EMS Lack of a meaningful world. Another example is Dissociative Identity Disorder, which is characterized by the experience of the self as consisting of separated, fully dissociated parts, as is most probably expressed by high levels of the EMS Lack of a coherent identity. Still another example is Schizotypal Personality Disorder, where patients report extreme confusion about their inner world, as well as their outer world, as we expect is reflected by elevated scores on both EMSs within the domain of Self-Coherence. Therefore, these EMSs are a welcome addition to ST theory, as, in our view, they cover forms of psychopathology that were hitherto not encompassed by ST theory.

\section{The Need for Fairness}

Discussion in the workgroup revealed that still another important need was missing in Young's list: the need for fairness. Ethologists have discovered that monkeys, apes, dogs, and birds already have a need for fairness, for instance refusing food if they get clearly less (attractive) food than another animal (e.g., Brosnan and de Waal 2014). This observation 
is incompatible with a simple rational economic view where accepting any food is better than refusing food and therefore getting nothing. Hence, a need for fairness seems to play a role here. This need is assumed to promote cooperation with individually known partners, also in humans (e.g., Starmans et al. 2017). Clinical observations indicate that frustrating the need for fairness can lead to severe emotional problems, and empirical studies have documented associations between unfairness (inequality, injustice) and mental as well as physical health problems (e.g., Prilleltensky 2013), and demonstrated that the need for fairness is evident already early in childhood (e.g., McAuliffe et al. 2017).

One could argue that the need for fairness is just part of the need for trust, or predictability in Dweck's model, and the need for safety in Young's model. However, given the specificity of this need for species (including humans) that depend on individual cooperation, the specific triggers, and the specific primary response (protest, refusal, anger, cf. Brosnan and de Waal 2014), we felt that it would be useful to postulate it as a separate need. Note that the need for trust is more related to predictable social affiliation than to cooperation. Also, note that fairness implies predictability, but predictability does not imply fairness: unfair treatment can be highly predictable. Hence, we propose a core need for fairness, which, when frustrated, can lead to the development of an EMS of Unfairness - a fundamental representation of (a) the world (including but not necessarily restricted to other people) as being unfair and unjust, (b) the society as lacking justice, thus not correcting those that behave unfairly, and (c) the self as a (continuous) victim of unfairness.

The last aspect is essential, as it represents the emotional pain that is part of this EMS (see also Ellis and Ellis 2011). Activation of this schema will lead to feelings of indignation and anger combined with powerlessness. It might be difficult for people with this EMS to deal with even slight experiences of unfairness. It is expected that this EMS is characteristic for people who behave in a victimized way and easily feel resentment because of (perceived) unequal treatment between them and others.

After having discussed basic needs and based on that analysis having added three EMSs to the 18 proposed by Young et al. (2003), we will now discuss how different ways of dealing with activation of an EMS lead to different schema modes.

\section{Evaluation and Reformulation of Coping with EMS Activation}

\section{Coping as Formulated in the Original ST Theory}

As was delineated in the introduction, ST theory states that the way a person copes with the (threat of) activation of an
EMS leads to a state of feeling, thinking, and behaving that is called a schema mode. Three ways of (dysfunctional) coping are described: surrender, avoidance, and overcompensation. We now describe them step by step.

1. Surrendering to an EMS activation leads to a state of feeling, thinking, and behaving as if the EMS is true. Two main groups of schema modes can result. First, a dysfunctional child mode can result from yielding to an EMS activation: the person feels as if (s)he is a child in a world defined by the representation of the EMS. For instance, an EMS of Abandonment can lead to the Abandoned Child mode when surrender is the way of coping. In this mode, the person feels the panic and desolation that is normal and functional for a little child that is abandoned, and which creates a strong drive to restore the connection with the caregiver. Second, if the EMS is based on the internalization of moral or achievement values, an internalized parent mode can result. For instance, surrendering to the EMS Punitiveness can lead to activation of the Punitive Parent Mode.

2. Avoidance of (full) EMS activation leads to the avoidance strategy dominating the person's state. Several avoidant coping modes have been described, e.g. the Detached Protector (detaching from emotions, needs, and beliefs associated with the EMS, resulting in a robot-like state), and the Self-Soother (actively engaging in behavior that soothes the emotional pain associated with the EMS).

3. Overcompensation of an EMS leads to a state in which the opposite of the EMS is felt and believed, while the behavior serves to prove this opposite. Perhaps the best known example is that of the Self-Aggrandizer, which overcompensates EMSs such as Failure and/or Emotional Deprivation.

As far as we know, two studies have tested the model proposing that the way of handling EMS activation mediates the relationship between EMSs and schema modes. Both studies used a newly developed Schema Coping Inventory (Rijkeboer et al. 2010), to assess the way of coping that people tend to use when confronted with an EMS activation. In the first study, Rijkeboer and Lobbestael (2012) did formal mediation tests on a large data set consisting of adult patient respondents $(\mathrm{N}=1602)$, splitting the sample in two for cross-validation purposes. In short, clear evidence was found for the mediating role of coping responses in the relationship between specific EMSs and schema modes for almost every combination tested (explained variance ranged from 0.34 to 0.74 ).

The second study was conducted on data of a mixed patient and non-patient sample of 699 adolescents (van Wijk-Herbrink et al. 2018a, b). To reduce the number of 
tests, only EMSs of the first domain of disconnection and rejection were investigated. The study found evidence for the relationship between first domain EMSs and Vulnerable Child mode being mediated by surrender coping, the relationship between first domain EMSs and Detached Protector mode being mediated by avoidance coping, and the relationship between first domain EMSs and Angry Child mode being mediated by overcompensating coping.

Thus, findings seem to corroborate the theory as originally formulated by Young et al. (2003).

Yet, discussion in the workgroup revealed a problem with both studies, that is, the Schema Coping Inventory had a subscale for overcompensation that by hindsight seems to assess externalization (thus a fight response), highlighting the confusion that exists around the definition of the ways of coping in ST theory. We will elaborate on this below.

\section{Coping with Internal Triggers Versus External Threats}

Young derived the three ways of coping with EMS activation as psychological analogues of basic survival responses in animals when faced with a severe threat (i.e., attack): fight (cf., overcompensation), flight (cf., avoidance), and freeze (cf., surrender). However, there is a loose relationship between these innate defense responses against acute external threats and the mental operations that people can employ to deal with EMS activation, which is confusing.

In schema theory, we need to describe how people handle EMS activation, which primarily has an intrapsychic function, and not a function to deal with external threats. Nevertheless, external circumstances can trigger an EMS, which through the way a person handles this activated EMS, leads to a schema mode that includes behavioral responses. Such behavioral responses might involve behaviors directed at the external circumstances, and as such can also include coping with the external situation. However, because it is the EMS that gives meaning to the external situation, the coping we describe here is primarily directed towards the EMS. In other words, the coping with the external situation serves the coping with the (threatening) EMS activation. For example, in an abandonment situation, aggressively threatening the other to not leave the person, serves to prevent the unbearable situation that being abandoned means according to the Abandonment EMS. Thus, it is ultimately the EMS towards which the coping is directed.

Therefore, we stress that the term coping in ST theory refers to handling EMS activation. In this paper we use the terms coping, handling, and dealing with EMS activation interchangeably.

\section{Evaluating the Labels of the Ways of Coping with EMS Activation}

The terms overcompensation and surrender also create confusion. The suggestion that overcompensation is the psychic equivalent of fighting an attacker, and surrender the psychic equivalent of submission to an attacker, has created confusion between (a) the type of response including its associated emotion (fight and anger, hence aggression when overcompensating; submission and fear when surrendering), and (b) the mental function of "overcompensation" and "surrender" (denying the EMS by believing the opposite, respectively believing the EMS). For instance, some people take the position that any angry or other externalizing behavior is the result of overcompensation (e.g., the so-called Angry Child mode is viewed as the result of overcompensating an EMS), as there are resistance and protest. On the other hand, others see all child modes, including the externalizing child modes, as resulting from surrendering to the EMS activation, as the EMS is believed to be true. Admittedly, with the last position it feels strange that surrender can result in a state of anger and protest. Moreover, with the position that overcompensation is characterized by fighting, hence anger, it is difficult to think of any overcompensation of EMS such as Entitlement, Unrelenting Standards, and Punitiveness.

Therefore, the workgroup proposes to use the following terms for the three ways of (maladaptive) handling EMS activation, with the aim to underline the intrapsychic function they have:

1. Resignation instead of surrender to describe the way of schema handling where the person gives in to the EMS activation, and fully believes the EMS.

2. Avoidance for the mental strategies characterized by avoiding or escaping EMS activation.

3. Inversion instead overcompensation to delineate the mental strategies where the person deals with EMS activation by believing the opposite of the EMS is true.

Please note that schema activation is not a dichotomous, but a dimensional issue. When a schema is activated to a certain extent, people can deal with it in a healthy adult way, which leads to healthy modes such as the Healthy Adult. Notwithstanding the importance of healthy handling EMS activation and the accompanying healthy modes, we decided to restrict ourselves in this paper to maladaptive mental strategies of dealing with EMS activation and the resulting unhealthy modes. 


\section{Towards a Comprehensive Taxonomy of Modes}

\section{Aspects of Modes}

Figure 2 is a diagrammatical representation of the model, where a trigger leads to the (threat of) activation of an EMS, dealt with by a specific way of coping, resulting in the listed types of schema modes. In addition to its function and motivation each schema mode has a cognitive, emotional, and behavioral element:

1. Cognitive content: the EMS is true (resignation), the content of the EMS is cognitively avoided (avoidance), or the opposite of the EMS is believed (inversion).

2. Feelings: the feeling is dictated by the EMS (resignation), the feeling is avoided (avoidance), or the feeling is the opposite of what would be expected with the EMS (inversion).

3. Behaviors: the behavior fits with the EMS being true (resignation), the behavior serves as protection for the person against full EMS activation (avoidance), or the behavior serves to prove that the opposite of the EMS is true (inversion).
Note, that in our view the Punitive Parent and the Demanding Parent are both the result of resignation to the activation of the EMS Punitiveness and Unrelenting Standards respectively.

The same accounts for the child modes; resignation to an activated schema, e.g., Abandonment/Instability EMS leads to an Abandoned Child mode. One regresses towards a state that is normal for a child in specific emotional circumstances, but for an adult this is dysfunctional.

\section{A Reformulated Theory; Some Examples}

Let us apply this model to a few EMSs. In the examples some possible schema modes are discussed rather than all possible modes (for a complete overview, see Appendix A).

Abandonment/Instability. A typical trigger of this EMS is a sign that the partner or another attachment figure is leaving (not necessarily abandoning) the person.

1. Resignation. The person believes it is true that the other is abandoning him/her, and that this is extremely threatening, as if the person were a little child left alone by the caregiver. Depending on his/her temperament, the person might become extremely anxious and upset, and start to cry and beg the other not to leave (abandonment panic) — or the person might become angry, accuse the other and aggressively block the way so that the other
Fig. 2 The reformulated schema mode model. A trigger leads to (threat of) EMS activation, which is dealt with by the person using a specific way of coping: resignation, avoidance, or inversion. This leads to the activation of specific schema modes, defined by function/ motivation, cognitive content, feelings, and behaviors

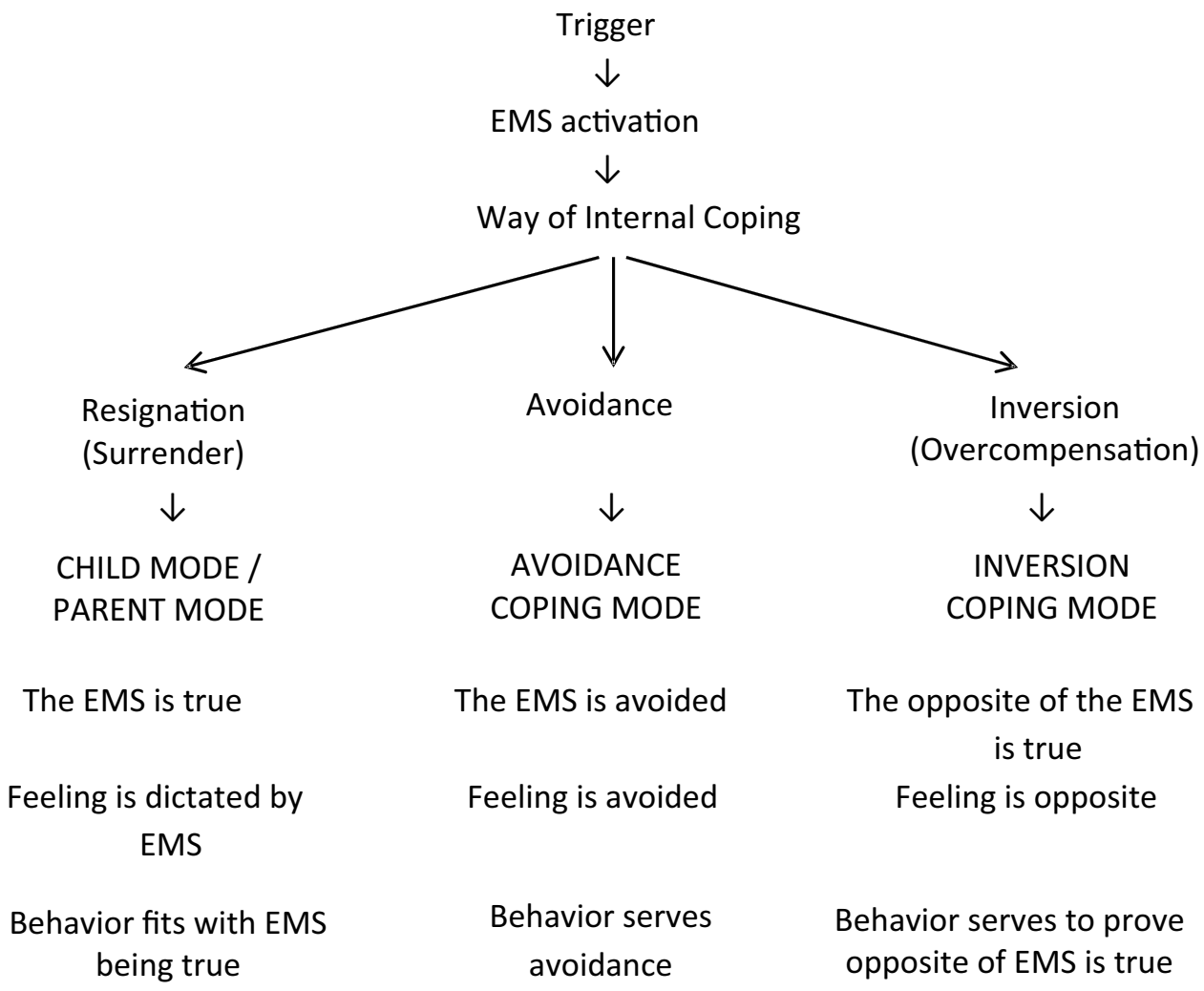

rigger

ctivation

ternal Coping

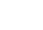

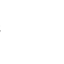


person cannot leave (frantic efforts to prevent abandonment). With the first response, we see an internalizing kind of response, resulting in activation of the Abandoned Child mode. The person shifts into a state of a little child panicking about being left alone. With the second response, we see an Angry Child response, in which the person desperately fights for not being abandoned. In both, the person believes that abandonment is taking place and feels that it is the end of the world.

2. Avoidance. The person might use massive detachment from the alarming signals that abandonment is going to take place by shutting off any connection to feelings and needs. The person might engage in distracting activities, or do nothing at all (e.g., lie in bed) and stop thinking to prevent that any triggers will activate the EMS and its associated emotions. The resulting coping mode is the Detached Protector.

3. Inversion. The person might deceive him/herself into believing they are unaffected by attachment, or loss of attachment, and act as if he or she does not need anybody. The feelings are dominated by independence, control, and liberty. The behavior is in line with this, e.g., the person leaves the other in a matter of fact way, displaying that (s)he is not attached to the other and does not need anybody. This is a mode that we propose to call the Hyper-Autonomous mode.

\section{Entitlement}

1. Resignation. The person believes it is true that (s)he is entitled and superior to others. Following Young's theorizing, typically a child-like state will follow. Thus, the belief of superiority and entitlement appears childlike, as do the feelings of pride experienced by the person. The behavior is inappropriate in its shameless display of superiority and entitlement, making it similarly childish (e.g., acts out childlike fantasies with fancy luxurious objects such as posh cars and grand houses). This would be the mode of a Grandiose or a Spoiled Child.

2. Avoidance. The person might use massive detachment from any signals that the EMS is activated. This comes at the price of detaching from compliments and recognition for achievements. The resulting coping mode is the Detached Protector.

3. Inversion. The person might deceive him/herself from thinking they are special into thinking (s)he is insignificant. The person believes (s)he does not deserve recognition for any achievement. The feelings are dominated by modesty and the behavior characterized by over-humbleness and downplaying personal importance. This is a mode that we propose to call the Overly-Humble.

\section{Unfairness}

1. Resignation. The person believes it is true that (s)he is treated unfairly, not only at a specific moment, but systematically, so that the balance is fundamentally upset. The belief in this injustice is difficult to follow for others. The person thus feels systematically unfairly treated and at the same time powerless. The person is complaining about being treated unfairly, indirectly expresses grudge, and easily takes the "victim role". This would be the mode of the Victimized Child. The person might also get angry and accuse others of treating them unfairly, hence an Angry Child arises.

2. Avoidance. The person might use massive detachment from any signals that the EMS is activated. The resulting coping mode is the Detached Protector.

3. Inversion. The person might deceive him/herself of the underlying pain of the EMS of Unfairness by taking pride in treating other people unfairly. This gives a powerful, dominant feeling, with sometimes sadistic elements. Instead of acting as a victim, the person takes the role of perpetrator. This would be the Bully-and-Attack mode. Another inversion would be the naïve belief that the world is fair, which would result in an overly trustful mode, which we call the Idealizer.

\section{A Comprehensive Taxonomy of Modes}

Note, that systematically combining all EMSs with the three ways of coping leads to 21 (EMSs) X $3=63$ schema modes. This number is even larger, because different subtypes of the resulting modes have been described. For instance, the type of child mode resulting from the EMS activation might be influenced by the temperament and the primary emotion that is triggered. Resignation to e.g., the Abandonment/ Instability EMS might lead to activation of the Abandoned Child mode, the Angry Child mode, or the Impulsive child mode. Thus, factors that might be related to trait (e.g., temperament (internalizing vs. externalizing)) or to state (e.g., substance use, perceived control over the other, et cetera) influence what type of child mode is activated. Note, that within the same person there might be a succession of child modes (e.g., the patient with borderline personality disorder switching from Abandoned to Angry Child and vice versa).

Similarly, within the group of avoidance modes several forms have been described and some of them empirically tested, such as the Detached Protector, the Self-Soother, the Avoidant Protector, and the Compliant Surrender (Bamelis et al. 2011; Lobbestael et al. 2008, 2010). Likewise, different forms of inversion modes have been proposed and some of them tested.

In sum, this would lead to an even larger number of schema modes than 63 . On the other hand, many coping 
modes are the same for each EMS, as they do not depend on the specific content of EMSs, but are characterized by the typical way of dealing with EMS activation (Young et al. 2003). Similarly, we argue that many child modes are variations of a similar state. The Angry Child mode, for instance, might vary in cognitive content depending on what EMS underlies it, the feelings and behaviors might cut across the different EMSs.

With these considerations in mind, the workgroup has systematically applied the model to all EMSs; the resulting modes are described in Appendix A. This appendix focuses on distinguishing the various dysfunctional modes, while distinguishing the various functional modes was not attempted.

\section{Reducing the Number of Modes}

Next, the number of modes was reduced to 40 by the following decisions (see Table 2 for the list of proposed modes):

1. The avoidance coping modes were formulated independently of EMS, as the coping dominates the feelings and thoughts, and not the EMS. This resulted in eight avoidance coping modes.

2. The vulnerable child modes were grouped together by the emotional need that relates to the EMSs. This means that seven vulnerable child modes resulted.

3. The child modes resulting from resignation to the two EMSs in the Realistic Limits \& Self-Control domain remained formulated as four separate (externalizing) child modes, as their phenomenology was so different.

4. The Angry Child modes were grouped into four types, depending on the type of anger and behavioral expression.

5. The parental or norm-setting modes were both retained, because of their different intentionality. Thus, Punisher and Demanding Critic modes are distinguished (formerly Punitive Parent and Demanding Parent; since norms can be set by other people as well, we refrained from using 'parent').

6. The inversion modes were formulated as six modes that are each related to several EMSs, and nine modes that are specific to one or two EMSs.

\section{Hypotheses on Externalizing Child Modes}

It is important to note that the model sketched above was not supported by all members of the workgroup. Some members take the position that externalizing child modes are the result of a rudimentary inversion (overcompensating) response to EMS activation. In short, they feel that in case of externalizing child modes, the initial response to the activation of most EMSs is a (short) activation of the vulnerable child mode after which the person fights the difficult to bear feelings by switching to an externalizing child mode. The workgroup agreed to make this an empirical issue. Hence, the following mediation models were hypothesized:

1. Moderated mediation: the relationship between vulnerable EMSs and externalizing child modes is mediated by resignation, with the mediation being moderated by externalizing temperament. For instance, the relationship between the EMS of Abandonment and Angry Child is mediated by resignation, and moderated by externalizing temperament (see Fig. 3).

Similarly, the relationship between vulnerable EMSs and internalizing child modes is mediated by resignation, with the mediation being moderated by internalizing temperament. For instance, the relationship between the EMS of Abandonment and Vulnerable Child mode is mediated by resignation, and moderated by internalizing temperament (see Fig. 4).

2. Double mediation: the relationship between vulnerable EMSs and externalizing versus internalizing child modes is mediated by resignation and temperament (see Fig. 5).

3. Mediation by different ways of coping: the relationship between vulnerable EMSs and externalizing versus internalizing child modes is mediated by different ways of coping. That is, inversion mediates the relationship with the Angry Child mode, and resignation that with the Vulnerable Child mode. Figure 6 shows these different mediation models for Abandonment EMS and the type of child modes.

In the next step of the project new inventories will be developed and data will be gathered in over 30 countries worldwide. This will enable the cross-cultural validation of all schema therapy related concepts, as well as a critical test of the different models concerning the relations between the various concepts.

\section{Discussion \& Conclusion}

In the last decades the application of schema therapy has evolved considerably; a broader range of disorders is treated in an increasing number of cultures worldwide and the focus has shifted gradually to mode models. Consequently, there is an urgent need for a cross-cultural valid and comprehensive taxonomy of modes. For that, an international workgroup was formed. A systematic approach was chosen, critically 
Table 2 Overview of proposed dysfunctional schema modes

\begin{tabular}{|c|c|}
\hline Schema mode $(\mathrm{N}=$ new $)$ & Respective EMS or need domain (in italic) \\
\hline \multicolumn{2}{|l|}{ Child modes } \\
\hline Disregarded child $(\mathrm{N})$ & EMSs in the domain Safety \& Nurturance \\
\hline Non-autonomous child (N) & EMSs in the domain Autonomy, Competence, \& Identity \\
\hline Subordinate child $(\mathrm{N})$ & EMSs in the domain Freedom to Express Opinions, \& Emotions \\
\hline Constrained child $(\mathrm{N})$ & EMSs in the domain Spontaneity \& Play \\
\hline Confused child $(\mathrm{N})^{\mathrm{a}}$ & EMSs in the domain Self-Coherence \\
\hline Over-diligent child (N) & Unrelenting standards \\
\hline Victimized child $(\mathrm{N})$ & Unfairness \\
\hline Grandiose child & Entitlement \\
\hline Spoiled child (N) & Entitlement \\
\hline Undisciplined child & Insufficient self-control/self-discipline \\
\hline Impulsive child & Insufficient self-control/self-discipline \\
\hline Angry child & Many EMSs \\
\hline Enraged child & Many EMSs \\
\hline Rebellious child (N) & Many EMSs \\
\hline Sulking child $(\mathrm{N})$ & Many EMSs \\
\hline \multicolumn{2}{|c|}{ Norm-setting modes (parental modes) } \\
\hline Demanding critic & Unrelenting standards \\
\hline Punisher & Punitiveness \\
\hline \multicolumn{2}{|l|}{ Avoidance modes } \\
\hline Detached protector & Nearly all EMSs \\
\hline Funny protector $(\mathrm{N})$ & Nearly all EMSs \\
\hline Angry protector & Nearly all EMSs \\
\hline Avoidant protector $(\mathrm{N})$ & Nearly all EMSs \\
\hline Compliant surrender & Nearly all EMSs \\
\hline Reassurance seeker $(\mathrm{N})$ & Nearly all EMSs \\
\hline Detached self-soother & Nearly all EMSs \\
\hline Suspicious overcontroller & Nearly all EMSs \\
\hline \multicolumn{2}{|l|}{ Inversion modes } \\
\hline Hyper-autonomous mode $(\mathrm{N})$ & $\begin{array}{l}\text { Abandonment, dependence/incompetence, Enmeshment, subjugation, self-sacri- } \\
\text { fice, approval seeking }\end{array}$ \\
\hline Clown $(\mathrm{N})$ & Nearly all EMSs \\
\hline Attention \& approval seeker & Emotional deprivation, defectiveness/shame, social isolation, emotional inhibition \\
\hline Self-aggrandizer & Nearly all EMSs \\
\hline Bully \& attack & Abandonment, subjugation, unfairness, mistrust/abuse \\
\hline Perfectionistic overcontroller & Failure, emotional deprivation, insufficient self-control \\
\hline Idealizer (N) & Mistrust/abuse \\
\hline Daredevil $(\mathrm{N})^{\mathrm{b}}$ & Vulnerability to harm \& illness \\
\hline Slacker/Oblomov $(\mathrm{N})^{\mathrm{c}}$ & Unrelenting standards \\
\hline Pollyanna/over-optimist $(\mathrm{N})^{\mathrm{d}}$ & Negativity/pessimism \\
\hline The merciful $(\mathrm{N})$ & Punitiveness \\
\hline The over-humble $(\mathrm{N})$ & Entitlement \\
\hline The pretender $(\mathrm{N})$ & Lack of a coherent identity, lack of meaningful world \\
\hline Conning \& manipulation $(\mathrm{N})$ & Abandonment, unfairness \\
\hline Predator & Unfairness \\
\hline
\end{tabular}

${ }^{a}$ The alternative, that a Confused Child mode (EMS Lack of Coherent Identity) and a Disconnected Child mode (EMS Lack of a Meaningful World) should be distinguished is an empirical issue. Here we propose one child mode covering both EMSs for reasons of sparsity

${ }^{b}$ Daredevil: A reckless person who enjoys doing dangerous things (Oxford dictionary)

${ }^{c}$ Oblomov: A sluggish, weak-willed, or procrastinating person (Oxford dictionary)

${ }^{\mathrm{d} P o l l y a n n a: ~ A n ~ e x c e s s i v e l y ~ c h e e r f u l ~ o r ~ o p t i m i s t i c ~ p e r s o n ~(O x f o r d ~ d i c t i o n a r y) ~}$ 


\section{EMS (e.g., Abandonment) $\longrightarrow$ Resignation $\longrightarrow$ Angry Child \\ Externalizing Temperament}

Fig. 3 Moderated mediation as a hypothesis of the relationship between vulnerable EMSs and the angry child mode

Fig. 4 Moderated mediation as a hypothesis of the relationship between vulnerable EMSs and the vulnerable child mode

\author{
EMS (e.g., Abandonment) $\longrightarrow$ Resignation $\longrightarrow \uparrow$ Vulnerable Child \\ Internalizing Temperament
}

EMS (e.g., Abandonment) $\longrightarrow$ Resignation $\longrightarrow$ Externalizing $\longrightarrow$ Angry Child

EMS (e.g., Abandonment) $\longrightarrow$ Resignation $\longrightarrow$ Internalizing $\longrightarrow$ Vulnerable Child

Fig. 5 Double mediation as a hypothesis of the relationship between vulnerable EMSs and either the angry or vulnerable child modes

EMS (e.g., Abandonment) $\longrightarrow$ Inversion $\longrightarrow$ Angry Child

EMS (e.g., Abandonment) $\longrightarrow$ Resignation $\longrightarrow$ Vulnerable Child

Fig. 6 Different mediation as a hypothesis of the relationship between vulnerable EMSs and either the angry or vulnerable child modes

evaluating and updating the theory underlying ST, resulting in the present position paper.

Reviewing the literature two omissions were discovered in the original formulation of the theory, related to two core needs: self-coherence and fairness. On the basis of these additional needs, three new EMSs are proposed: Lack of a Coherent Identity, Lack of a Meaningful World, and Unfairness. Next, the workgroup reflected on the three ways of dealing with EMS-activation stipulated by Young et al. (2003), which highlighted the confusion surrounding these concepts. Therefore, we relabeled two of the three ways of maladaptive coping with schema activation, so that the focus was put on their function; that is, dealing with intrapsychic processes related to schema activation, and not with external threat. We tentatively labeled these two ways of maladaptive coping as resignation (formerly: surrender), and inversion (formerly: overcompensation). Hereafter, the workgroup derived schema modes by systematically combining each EMS with each way of handling schema activation, leading to over 63 schema modes, which we next reduced, by combining some of the modes within each need domain, to a list of 40 modes. Note that during this initial theoretical process, different views were expressed in the workgroup about the mediation of the relationship between externalizing child modes and EMSs by coping with EMS activation. Given a lack of consensus we developed different mediation models that we will test during the empirical phase of the project.

This project has some advantages compared to previous methods to develop an instrument for assessing schema modes. First, the hypothesized modes are derived on the basis of a clear theoretical framework. This will help to achieve a more comprehensive coverage of relevant schema modes that are manifested in patients. It also sets the ground for testing the theoretical views on the relationship between EMSs, ways of coping, and schema modes, as well as examining the newly formulated EMSs, especially whether both EMSs that stem from the need of Self-Coherence are distinguishable constructs. Second, we opted for an international approach from the outset. The proposed modes will form the basis of the construction of a new Schema Mode 
Inventory. Item writing and tests will be done in parallel in multiple countries $(>30)$ and languages $(>23)$, thus we expect to develop a cross-culturally valid instrument. The planned empirical studies also allow to test several models of the relationship between EMS, coping, and schema modes, which will contribute to the theory underlying ST.

Supplementary Information The online version contains supplementary material available at https://doi.org/10.1007/s10608-021-10209-5.

Acknowledgements We thank Eshkol Rafaeli for his valuable comments on earlier drafts of this paper.

\section{Compliance with Ethical Standards}

Conflict of Interest Arnoud Arntz, Marleen Rijkeboer, Edward Chan, Eva Fassbinder, Alp Karaosmanoglu, Christopher William Lee, Marta Panzeri declare that they have no conflict of interest.

Open Access This article is licensed under a Creative Commons Attribution 4.0 International License, which permits use, sharing, adaptation, distribution and reproduction in any medium or format, as long as you give appropriate credit to the original author(s) and the source, provide a link to the Creative Commons licence, and indicate if changes were made. The images or other third party material in this article are included in the article's Creative Commons licence, unless indicated otherwise in a credit line to the material. If material is not included in the article's Creative Commons licence and your intended use is not permitted by statutory regulation or exceeds the permitted use, you will need to obtain permission directly from the copyright holder. To view a copy of this licence, visit http://creativecommons.org/licenses/by/4.0/.

\section{References}

Arntz, A., \& Jacob, G. (2012). Schema therapy in practice. Chichester: Wiley.

Arntz, A., \& van Genderen, H. (2009). Schema therapy for borderline personality disorder. Chichester: Wiley.

Ayyash-Abdo, H., Tayara, R., \& Sasagawa, S. (2016). Social anxiety symptoms: A cross- cultural study between Lebanon and the UK. Personality and Individual Differences, 96, 100-105. https://doi. org/10.1016/j.paid.2016.02.079.

Bamelis, L. L. M., Renner, F., Heidkamp, D., \& Arntz, A. (2011). Extended schema mode conceptualizations for specific personality disorders: An empirical study. Journal of Personality Disorders, 25, 41-58. https://doi.org/10.1521/pedi.2011.25.1.41.

Baumeister, R. F., \& Leary, M. R. (1995). The need to belong: Desire for interpersonal attachments as a fundamental human motivation. Psychological Bulletin, 117, 497.

Brosnan, S. F., \& de Waal, F. B. (2014). Evolution of responses to (un) fairness. Science, 346(6207), 1251776. https://doi.org/10.1126/ science. 1251776.

Chan, E., \& Tan, H. J. (2020). Positive psychology couple schema therapy: A new model of couple therapy focusing on reigniting couple attraction via schema therapy and positive psychology. Journal for ReAttach Therapy and Developmental Diversities, 22, 61-69. https://doi.org/10.26407/2019jrtdd.1.24.

de Klerk, N., Abma, T. A., Bamelis, L. L., \& Arntz, A. (2017). Schema therapy for personality disorders: A qualitative study of patients' and therapists' perspectives. Behavioural and Cognitive
Psychotherapy, 45, 31-45. https://doi.org/10.1017/S135246581 6000357.

Deci, E. L., \& Ryan, R. M. (2000). The "what" and "why" of goal pursuits: Human needs and the self-determination of behavior. Psychological Inquiry, 11, 227-268.

Dweck, C. S. (2017). From needs to goals and representations: Foundations for a unified theory of motivation, personality and development. Psychological Review, 124, 689-719. https://doi. org/10.1037/rev0000082.

Ellis, A., \& Ellis, D. J. (2011). Theories of psychotherapy. Rational emotive behavior therapy. Washington, D.C.: American Psychological Association.

Hofmann, S. G. (2006). The importance of culture in cognitive and behavioral practice. Cognitive and Behavioral Practice, 13, 243 255. https://doi.org/10.1016/j.cbpra.2006.07.001.

Jacob, G. A., \& Arntz, A. (2013). Schema therapy for personality disorders-A review. International Journal of Cognitive Therapy, 6, 171-185.

Keulen-de Vos, M. E., Bernstein, D. P., Clark, L. A., de Vogel, V., Bogaerts, S., Slaats, M., \& Arntz, A. (2017). Validation of the schema mode concept in personality disordered offenders. Legal and Criminological Psychology, 22, 420-441. https://doi. org/10.1111/lcrp.12109.

Keulen-de Vos, M. E., Bernstein, D. P., Vanstipelen, S., de Vogel, V., Lucker, T. P. C., Slaats, M., et al. (2016). Schema modes in criminal and violent behaviour of forensic cluster B PD patients: A retrospective and prospective study. Legal and Criminological Psychology, 21, 56-76. https://doi.org/10.1111/lcrp.12047.

Lobbestael, J., van Vreeswijk, M., \& Arntz, A. (2007). Shedding light on schema modes: A clarification of the mode concept and its current research status. Netherlands Journal of Psychology, 63, 76-85. https://doi.org/10.1007/BF03061068.

Lobbestael, J., van Vreeswijk, M., \& Arntz, A. (2008). An empirical test of schema mode conceptualizations in personality disorders. Behaviour Research and Therapy, 46, 854-860. https://doi. org/10.1016/j.brat.2008.03.006.

Lobbestael, J., van Vreeswijk, M., Spinhoven, P., Schouten, E., \& Arntz, A. (2010). Reliability and validity of the Short Schema Mode Inventory (SMI). Behavioural and Cognitive Psychotherapy, 38, 437-458. https://doi.org/10.1017/S1352465810000226.

McAuliffe, K., Blake, P. R., Steinbeis, N., \& Warneken, F. (2017). The developmental foundations of human fairness. Nature Human Behaviour, 1, 0042.

Pittman, T. S., \& Zeigler, K. R. (2007). Basic human needs. In A. W. Kruglanski \& E. T. Higgins (Eds.), Social psychology: Handbook of basic principles (2nd ed., pp. 473-489). New York, NY: Guilford Press.

Prilleltensky, I. (2013). Wellness without fairness: The missing link in psychology. South African Journal of Psychology, 43, 147-155. https://doi.org/10.1177/0081246313484238.

Rafaeli, E., Bernstein, D. P., \& Young, J. (2010). Schema therapy: Distinctive features. Abingdon: Routledge.

Renner, F., Arntz, A., Leeuw, I., \& Huibers, M. (2013). Treatment for chronic depression using schema therapy. Clinical Psychology: Science and Practice, 20, 166-180. https://doi.org/10.1111/ cpsp.12032.

Rijkeboer, M. M., \& Lobbestael, J. (2012). The relationships between early maladaptive schemas, schema modes, and coping styles: An empirical study. In M. M. Rijkeboer (Ed.), Assessment of schema concepts and their interrelationships [Symposium]. New York, USA: 5th World Conference of Schema Therapy.

Rijkeboer, M. M., Lobbestael, J., Arntz, A., \& van Genderen, H. (2010). Schema coping inventory. Utrecht: Universiteit Utrecht.

Sempérteguia, G. A., Karremana, A., Arntz, A., \& Bekker, M. H. J. (2013). Schema therapy for borderline personality disorder: A comprehensive review of its empirical foundations, effectiveness 
and implementation possibilities. Clinical Psychological Review, 33, 426-447. https://doi.org/10.1016/j.cpr.2012.11.006.

Simpson, S. G., Morrow, E., van Vreeswijk, M., \& Reid, C. (2010). Group schema therapy for eating disorders: A pilot study. Frontiers in Psychology, 1, 182. https://doi.org/10.3389/fpsyg .2010 .00182 .

Starmans, C., Sheskin, M., \& Bloom, P. (2017). Why people prefer unequal societies. Nature Human Behaviour, 1, 0082.

Tan, Y. M., Lee, C. W., Averbeck, L. E., Brand-de Wilde, O., Farrell, J., Fassbinder, E., ... \& Arntz, A. (2018). Schema therapy for borderline personality disorder: A qualitative study of patients' perceptions. PloS One, 13, e0206039.https://doi.org/10.1371/ journal.pone.0206039

van Wijk-Herbrink, M. F., Bernstein, D. P., Broers, N. J., Roelofs, J., Rijkeboer, M. M., \& Arntz, A. (2018b). Internalizing and externalizing behaviors share a common predictor: The effects of early maladaptive schemas are mediated by coping responses and schema modes. Journal of Abnormal Child Psychology, 46, 907-920. https://doi.org/10.1007/s10802-017-0386-2. van Wijk-Herbrink, M. F., Roelofs, J., Broers, N. J., Rijkeboer, M. M., Arntz, A., \& Bernstein, D. P. (2018a). Validation of schema coping inventory and schema mode inventory in adolescents. Journal of Personality Disorders, 32, 220-241. https://doi.org/10.1521/ pedi_2017_31_295.

Young, J. E. (1994). Cognitive therapy for personality disorders: A schema-focused approach. Sarasota, FL: Professional Resource Press.

Young, J., Arntz, A., Atkinson, T., Lobbestael, J., Weishaar, M., van Vreeswijk, M., \& Klokman, J. (2007). Schema mode inventory. New York: Schema Therapy Institute.

Young, J. E., Klosko, J. S., \& Weishaar, M. E. (2003). Schema therapy: A practitioner's guide. New York: Guilford Press.

Publisher's Note Springer Nature remains neutral with regard to jurisdictional claims in published maps and institutional affiliations. 